\title{
Developmental plasticity in covariance structure of the skull: effects of prenatal stress
}

\author{
Paula N. Gonzalez, ${ }^{1}$ Benedikt Hallgrímsson ${ }^{2}$ and Evelia E. Oyhenart ${ }^{3}$ \\ ${ }^{1}$ CONICET, División Antropología, Facultad de Ciencias Naturales y Museo, Universidad Nacional de La Plata, La Plata, Argentina \\ ${ }^{2}$ Department of Cell Biology and Anatomy, University of Calgary, Calgary, AB, Canada \\ ${ }^{3}$ CONICET, Instituto de Genética Veterinaria, Facultad de Ciencias Veterinarias, UNLP-CCT La Plata, Argentina
}

\begin{abstract}
Environmental perturbations of many kinds influence growth and development. Little is known, however, about the influence of environmental factors on the patterns of phenotypic integration observed in complex morphological traits. We analyze the changes in phenotypic variance-covariance structure of the rat skull throughout the early postnatal ontogeny (from birth to weaning) and evaluate the effect of intrauterine growth retardation (IUGR) on this structure. Using 2D coordinates taken from lateral radiographs obtained every 4 days, from birth to 21 days old, we show that the pattern of covariance is temporally dynamic from birth to 21 days. The environmental perturbation provoked during pregnancy altered the skull growth, and reduced the mean size of the IUGR group. These environmental effects persisted throughout lactancy, when the mothers of both groups received a standard diet. More strikingly, the effect grew larger beyond this point. Altering environmental conditions did not affect all traits equally, as revealed by the low correlations between covariance matrices of treatments at the same age. Finally, we found that the IUGR treatment increased morphological integration as measured by the scaled variance of eigenvalues. This increase coincided and is likely related to an increase in morphological variance in this group. This result is expected if somatic growth is a major determinant of covariance structure of the skull. In summary, our findings suggest that environmental perturbations experienced in early ontogeny alter fundamental developmental processes and are an important factor in shaping the variance-covariance structure of complex phenotypic traits.
\end{abstract}

Key words developmental process; intrauterine growth retardation; nutritional stress; variance-covariance structure; Wistar rat.

\section{Introduction}

Morphological variation emerges from complex interactions between genetic and environmental factors that are modulated by sequential and interacting developmental processes (Fig. 1a). The importance of developmental interactions that mediate between genes and phenotypic traits has long been recognized by developmental biologists. Developmental processes can be viewed as acting at many hierarchically arranged levels from the molecular and developmental-genetic to the interaction of organs and the interactions between the organism and its environment (Hall, 2003). At the cellular level, for example, they include cell behaviors such as mitosis, apoptosis, migration, changes

Correspondence

Paula N. Gonzalez, División Antropología, Facultad de Ciencias Naturales y Museo, Paseo del Bosque S/N, La Plata (1900), Argentina. E: pgonzalez@fcnym.unlp.edu.ar; paulan.gonza@gmail.com

Accepted for publication 5 November 2010 in adhesive properties, and secretion of extracellular signals, among others, which result in a specific spatial distribution of cells and extracellular components (SalazarCiudad et al. 2003). Developmental processes at higher levels comprise functional interactions at the tissue and organ levels, fusion of morphological elements and somatic growth, among others (Hall, 2003; Hallgrímsson \& Lieberman, 2008). As a consequence of this hierarchical organization, one can identify developmental processes that are particularly relevant as determinants of some type of phenotypic variation. For example, the growth of the brain within the skull is a major determinant of craniofacial shape, but the set of developmental processes responsible for brain size are not necessarily relevant to account for skull shape (Hallgrímsson et al. 2007, 2009). The selection of a relevant scale for the phenomena under study is the main idea underlying the so-called 'middle-out approach', proposed as an alternative to approaches such as 'topdown' or 'bottom-up' that focus primarily on genotypephenotype relationships (Noble, 2002; Hallgrímsson \& Lieberman, 2008). 
A

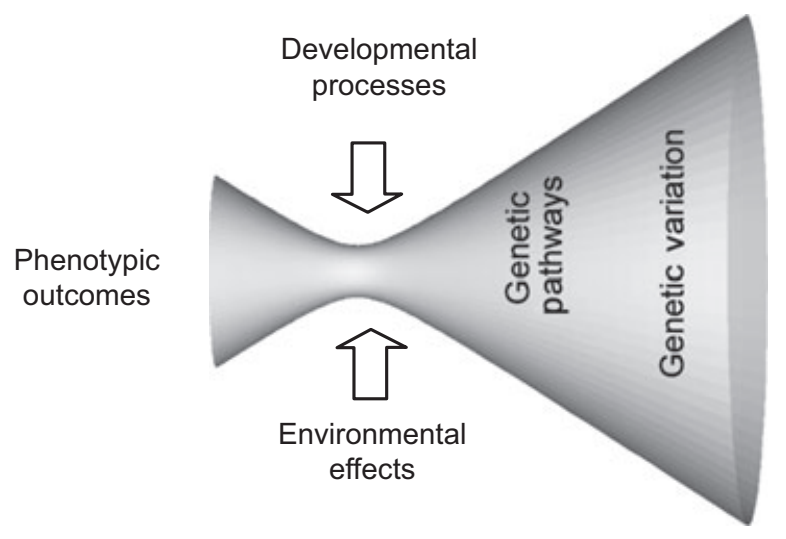

B

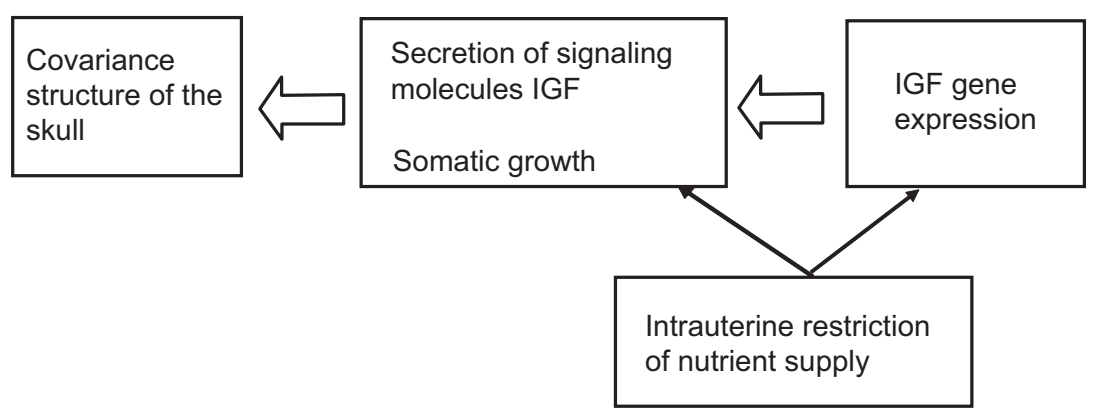

Fig. 1 (a) Conceptual framework about the origin of phenotypic variation during development. Genetic factors affect phenotypic traits through a set of developmental processes, which in turn are affected by internal and external environmental factors experienced by an organism in the course of its ontogeny. Modified from Hallgrímsson \& Lieberman (2008). (b) Relationship between the environmental factor under study intrauterine restriction in nutrient supply, the developmental processes affected and the resulting patterns of phenotypic variation in the skull. IGF, insulin-like growth factor.

The relative influence of the different developmental processes on phenotypic covariation structure changes in the course of ontogeny (Hallgrímsson et al. 2009; Mitteroecker \& Bookstein, 2009). Therefore, the ontogeny of any phenotypic trait can be described as a sequence of pattern transformations resulting from different developmental processes acting on previous patterns. In complex structures such as the skull, the patterns of phenotypic variancecovariance arise from the differential influence of developmental processes on particular traits as well as from the magnitude of their variances (Hallgrímsson et al. 2007). In other words, phenotypic variance-covariance structure is determined by the variance of covariation-generating developmental processes (Hallgrímsson et al. 2007, 2009; Mitteroecker \& Bookstein, 2007). That means that any genetic or environmental factor that increases the variance of one of these key developmental processes should also alter the covariance structure and the overall integration of morphological components. The direction of such changes depends on the relative importance of the process in determining covariance structure. If the variance of a process that is the major determinant of phenotypic covariance in a population is increased during ontogeny, either by genetic or environmental factors, then integration level is expected to increase; whereas if a developmental process that contributes very little to the covariance structure in a population increases dramatically in variance, the opposite would occur and integration will decrease (Hallgrímsson et al. 2007).

The factors that regulate developmental processes depend substantially on the environmental context. Although the proximate effects eventually feed into developmental-genetic pathways, the causality resides in the interplay between internal and external factors. Therefore, the environment experienced throughout ontogeny plays an important role in shaping the phenotypic traits observed at every age. This is particularly relevant for morphological traits, as their phenotypic plasticity is fundamentally a developmental phenomenon, i.e. developmental pathways are expressed differently in response to specific environmental factors to produce continuously varying traits or discrete, alternative phenotypes (Aubin-Horth \& Renn, 2009; Gilbert \& Epel, 2009).

Nutrition is a key factor in the regulation of fetal growth, in which the interaction among the fetus, the placenta and the maternal organism plays a very important role. Although nutrients are required for growth, in multicellular organisms they do not directly stimulate cell growth (Nijhout, 2003). Intrauterine and early postnatal nutritional 
changes translate into alterations of somatic growth because they affect most of the factors that regulate growth in the course of ontogeny (Bloomfield et al. 2006). Prenatal growth is mainly regulated by insulin-like growth factors (IGF-I and IGF-II). IGF-II is the primary factor involved in embryonic growth, whereas IGF-I, produced by the fetal liver and other tissues, is the dominant fetal growth regulator in late gestation (Allan et al. 2001; Le Roith et al. 2001). IGFs have insulin-like metabolic effects and stimulate cell proliferation and differentiation, and these mitogenic effects are mediated through interaction with the IGF receptor (IGF-IR) or insulin receptor (Le Roith et al. 1995). The central importance of IGF for normal growth has been conclusively illustrated by the severe growth retardation exhibited by mice in which the IGF-I, IGF-II or IGF-I receptor genes have been inactivated by homologous gene targeting (DeChiara et al. 1990). Several studies on nutritional regulation of IGF during prenatal growth have shown that nutrient restriction leads to reduction of fetal growth associated with reduced circulating and liver IGF-I levels and altered IGF-binding proteins (Martín et al. 2005).

The underlying molecular mechanisms that give rise to this reduction are not definitively understood, but emerging evidence suggests that epigenetic (chromatin modification) mechanisms might alter IGF-I levels after nutritional insult. Recently, Fu et al. (2009) found that intrauterine growth retardation (IUGR) modifies the histone code along the length of the hepatic IGF-I gene, and that many such changes persist postnatally. By contrast, serum IGF-II and liver IGF-II mRNA expression seem to be less affected by general food restriction (Fowden, 2003; Martín et al. 2005). Figure $1 \mathrm{~b}$ summarizes the relationship between the environmental factor analyzed here, the developmental processes involved and the resulting patterns of phenotypic variation in the skull. Previous studies that have evaluated the effect of malnutrition on the skull have focused on size and shape mean differences between control and undernourished animals (Dahinten \& Pucciarelli, 1986; Dressino \& Pucciarelli, 1996; Miller \& German, 1999; Oyhenart et al. 2003). However, to date no attempt has been made to assess these perturbations using variance-covariance matrices in order to evaluate the influence of environmental factors on the patterns of phenotypic integration observed.

This study aims to: (i) analyze the changes in phenotypic variance-covariance structure of the skull throughout early postnatal ontogeny (from birth to weaning); and (ii) evaluate the effect of environmental-induced changes in a specific development process, prenatal somatic growth, on this variance-covariance structure. We focus on prenatal growth because the effect of environmental factors depends closely on their timing in the organism's ontogeny (Badyaev, 2005; Sultan \& Stearns, 2005), and this is a particularly sensitive period in which the basic patterns for critical traits as well as the response of the organism to postnatal environmental factors are determined irreversibly (Gluckman \& Hanson,
2004). Particularly, we evaluated the following hypotheses: (i) if developmental processes that determine morphological variation are differentially expressed in the course of ontogeny, the variance-covariance structure of the skull will change with age and the similitude in such structure will be greater between closer age stages; (ii) if the alteration of somatic growth has the same effect on all morphological components of the skull, the experimentally induced changes in this developmental process will not alter the covariance structure of the skull; (iii) if somatic growth has an important influence on covariance structure, an increase in the variance of this developmental process should increase the phenotypic integration of the complex morphological traits studied.

\section{Materials and methods}

\section{Sample}

The animals employed in this study were Rattus norvegicus albinus, var. Wistar, brought from the Comisión Nacional de Energía Atómica in 1997. They were grown as an outbred colony in the animal house of the Instituto de Genética Veterinaria (IGEVET), for about 12 generations up to the experiment. The outbreeding condition was assured by maintaining a minimal stock of 200 rats free of experimentation. Periodic genetic monitoring was carried out to avoid bottlenecks or similar effects. The animals were kept free of pathogens and treated in compliance with standardized institutional guidelines. Rats were individually housed in solid stainless steel cages $\left(12^{\prime} \times 12^{\prime} \times 6.8^{\prime}\right)$, which were cleaned three times a week. The room temperature ranged from 21 to $25^{\circ} \mathrm{C}$, and the photoperiod was $12 \mathrm{~h}$ of light, from $06: 00$ hours to $18: 00$ hours. They were fed on a pelleted and sterilized commercial stock diet containing proteins $(23 \%)$, carbohydrates $(44 \%)$, lipids $(11 \%)$, water $(8 \%)$, fiber $(5 \%)$, mineral mixture $(3 \%)$ and vitamin mixture $(1 \%)$.

Fifty females (200-250 g body weight) were mated overnight with 10 adult males. Pregnancy was assumed to start after spermatozoa were found in the vaginal smear. Pregnant rats were housed in individual steel boxes, fed on stock diet and water ad libitum, and assigned to one of three experimental groups: (i) Control; (ii) IUGR; and (iii) Sham-operated (Sham).

Control dams did not receive any treatment. A lower midline laparotomy was performed in the mothers of the IUGR group at first day of gestation. Animals were anesthetized intramuscularly with Ketalar (Parke Davis; $0.005 \mathrm{~mL} 100 \mathrm{~g}^{-1}$ body weight). Complementary light-ether anesthesia was given during surgery. After opening the peritoneal cavity the uterus was exposed. The uterine vessels near the lower end of each uterine horn were bent and fastened with a 3-0 silk suture. Pregnancy was allowed to go on until delivery. The procedure applied to shamoperated animals was similar to that used for IUGR ones. The uterine vessels, however, were not obstructed in order to separate the effects of surgery from that of vessel bending.

IUGR and Sham pups were cross-fostered to control dams. Litters were reduced to four males and four females each, to make lactation uniform across the groups. Pups suckled ad libitum. Finally, the sample was composed as follows: (i) Control: 20 males and 18 females; (ii) IUGR: 16 males and 14 females; and (iii) Sham: 20 males and 15 females (Oyhenart et al. 2003). 


\section{Morphometric analyses}

Lateral radiographs of each animal were taken every 4 days, from birth to 21 days old. Then, the radiographs were digitized using a scanner (Epson Perfection V500). Craniofacial traits were captured from digital images as 2D coordinates for 11 landmarks and seven semi-landmarks in lateral view (Fig. 2; Table 1).

In order to estimate measurement error, we evaluated the intra-observer error associated with the placement of landmark coordinates. The experimental design devised to estimate the measurement error was made using a random sample of $30 \mathrm{X}$-rays composed by individuals of different ages and both sexes. Operator inconsistency was evaluated by digitizing the same set of points from images in two events 2 weeks apart from each other. We compared statistically the coordinates obtained in each series using the intra-class correlation coefficient (ICC) and repeated-measure ANOVA (ANOVARM). The results showed no significant levels of error in the placement of landmarks (with mean values of 0.97 for ICC,

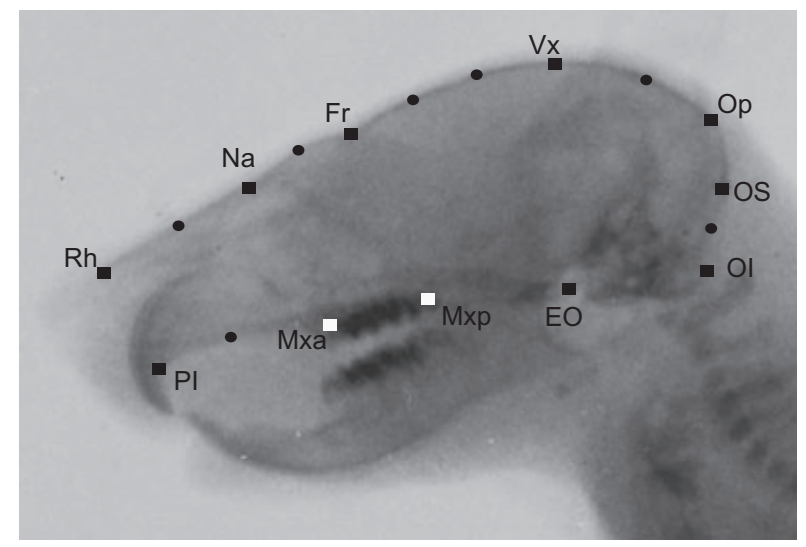

Fig. 2 2D landmarks (squares) and semi-landmarks (circles) collected from rat skulls from lateral view.

Table 1 List of 2D landmarks digitized from rat skulls.

\begin{tabular}{ll}
\hline Landmark & Definition \\
\hline $\mathrm{Rh}$ & $\begin{array}{c}\text { Rhinion. The anterior tip at the end of the } \\
\text { suture of the nasal bones } \\
\text { The mid-point of the frontal-nasal suture }\end{array}$ \\
$\mathrm{Na}$ & The narrowest point of the frontal bone \\
$\mathrm{Fr}$ & Vertex. Most superior point of the vault \\
$\mathrm{Vx}$ & Opistocranium. The most posterior point \\
$\mathrm{Op}$ & of the vault \\
$\mathrm{OS}$ & Most superior point of occipital foramen \\
$\mathrm{OI}$ & Most inferior point of occipital foramen \\
$\mathrm{SO}$ & Spheno-occipital synchondrosis \\
$\mathrm{MxP}$ & $\begin{array}{l}\text { Point located on the maxilla, posterior to } \\
\text { the last molar }\end{array}$ \\
$\mathrm{MxA}$ & $\begin{array}{l}\text { Point located on the maxilla, anterior to } \\
\text { the first molar }\end{array}$ \\
$\mathrm{PI}$ & The most anterior point on the maxillary \\
& alveolar process, between the central incisor \\
& teeth along the lingual side
\end{tabular}

and non-significant values for ANOVA-RM), except for the $x$ coordinate of landmark 4 . In consequence, this landmark was treated as a semi-landmark, following the procedure described below.

The 18 landmarks and semi-landmarks of the skull were aligned by means of Generalized Procrustes Superimposition (GPS; Rohlf \& Slice, 1990; Bookstein, 1997; Adams et al. 2004). This procedure optimally translates, scales and rotates coordinates of landmarks and semi-landmarks (Rohlf \& Slice, 1990). In addition, the semi-landmarks were slid along tangents to the outline of the curve until they minimize the Procrustes distances between corresponding points along the outline of the reference form, i.e. Procrustes average (Bookstein, 1997; Bookstein et al. 2002; Perez et al. 2006). The centroid size of the specimens (the square root of the summed squared distances from all landmarks and semi-landmarks to the configuration centroid) was measured for each data set and was used to scale the raw coordinates in the GPS (Bookstein, 1991).

Principal component analysis (Bookstein, 1991; Rohlf, 1993) was performed on superimposed coordinates of the 18 landmarks and semi-landmarks in order to describe the axis of major shape variation within and among samples. The principal components obtained from these variables are termed relative warps (RW) within geometric morphometrics. The shape changes with respect to the reference configuration (i.e. consensus form) associated with the major axis were visualized as deformation grids based on each RW axis (Rohlf, 1993). Individual ontogenetic trajectories were also studied in size-shape space, which was constructed as a principal component analysis of the Procrustes shape variables augmented by the natural logarithm of centroid size (Mitteroecker et al. 2004). These analyses were performed to evaluate the effect of the environmental perturbation on the individual trajectories, and the relation between ontogenetic trajectories and the variance-covariance structure in each treatment group.

The Procrustes shape coordinates of the 18 landmarks and semi-landmarks were employed to estimate covariance matrices. Because with 18 landmarks and semi-landmarks there are 32 degrees of freedom for the Procrustes shape coordinates, which are too many to estimate covariance matrices on only 30 specimens, we also performed a GPS with 11 landmarks (with 18 degree of freedom). In addition, the first 11 RW scores (with 11 degrees of freedom) from the analysis performed on superimposed coordinates of the 18 landmarks and semi-landmarks (accounting for $95 \%$ of the total shape variation) were used as shape variables.

In this study we used the tps series software (Rohlf, 2007) to record the coordinates of landmarks and semi-landmarks, superimpose the configurations, and calculate the centroid sizes and principal components.

\section{Phenotypic variance analysis}

Shape variances within ontogenetic stages by treatment were compared using Foote's (1993) disparity measurement. This is defined as morphological disparity $D=\sum\left(d_{i}^{2}\right) /(n-1)$, where $d_{i}$ represents the distance of the specimens to the group centroid. Disparity was measured using DISPARITYBox6 software (Sheets, 2003), which uses the partial Procrustes distance as $d_{i}$ measure. Confidence intervals of each disparity value were placed by bootstrapping, with replacement (900 Bootstrap; Sheets, 2003). 


\section{Phenotypic covariance structure analyses}

Total covariance matrices of 18 landmarks and semi-landmarks, 11 landmarks and $11 \mathrm{RW}$ scores were estimated for each treatment at the different ages. We did not use the pooled by sex covariance matrices because a MANOVA showed no significant shape differences between male and female individuals (results not shown). Nevertheless, we estimated the pooled by sex covariance matrices for 18 landmarks and semi-landmarks for each treatment at the different ages, and the pattern of covariance did not differ between total and pooled matrices (results not shown). The pooled within-group covariance matrix was estimated for these samples using a General Linear Model (Marroig \& Cheverud, 2001).

Phenotypic covariance structures were compared using matrix correlations (Marroig \& Cheverud, 2001) and the distance proposed by Mitteroecker \& Bookstein (2009) as the natural metric to compare covariance matrices, which is named here as MB. The matrix correlation is the Pearson correlation computed using the corresponding elements of the two matrices as paired observations and measure of the strength of association between them. A correlation of +1 indicates that the covariation patterns are equal or proportional. A correlation of zero indicates unrelated structure between the matrices, and a correlation of -1 specifies matrices that are mirror images (Roff, 2000; Marroig \& Cheverud, 2001). Significance of these correlations was assessed using Mantel's test (1000 replicates). Matrix correlations were then adjusted to account for small sample size following Marroig \& Cheverud (2001), using the formula $R_{\text {adj }}=$ $R_{\text {obs }} / R_{\text {max }}$. Maximum matrix correlation $\left(R_{\max }\right)$ was estimated using the formula $R_{\max }=\left(t_{\mathrm{a}} t_{\mathrm{b}}\right)^{1 / 2}$. To estimate covariance matrix repeatability $(t)$, the original datasets were resampled and covariance matrices re-estimated 1000 times, and the mean matrix correlation between these and the original datasets was taken as an estimate of $t$ (Marroig \& Cheverud, 2001). To compare correlations across age/treatment groups and evaluate if the differences between them are significant, we estimated the 95.0\% confidence interval for Mantel correlation by bootstrap resampling.

The MB distance between two covariance matrices is calculated as the square root of the summed squared logarithms of the relative eigenvalues between the two matrices (Mitteroecker \& Bookstein, 2009). Mitteroecker \& Bookstein (2009) pointed out that this is the natural metric on the space of positive-definite symmetric matrices. These are measures of similarity or distance, not significance tests, and more emphasis should be placed on these measures than on the tests of significance (Marroig \& Cheverud, 2001; Mitteroecker \& Bookstein, 2009). There is a large amount of evidence that cranial morphometric variables of mammals covary significantly, and then it is more interesting to investigate the pattern and magnitude of this covariation in relation to evolutionary and environmental factors than estimate their significance.

Finally, covariance matrices were also compared using a random skewers vector correlation (Cheverud, 1996) so that our results may be compared with previous studies. The method of random skewers computes the response of covariance matrices being compared with a random selection vector, and then compares these responses using the vector correlation between the paired expected responses (Cheverud, 1996). This vector correlation provides a measure of the similarity among covariance matrices. Vector correlations are 1 when matrices are identical or proportional, and are zero when matrices have unrelated structure.

The pattern of similarity and distance generated by matrix correlation, random skewers vector correlation and the MB distance were further compared using principal coordinate analysis (PCo). The distance between each pair of covariance matrices was defined as one minus the matrix or vector correlation between them. The ordinations generated for each metric were compared using Procrustes analysis (Gower, 1971; Peres-Neto \& Jackson, 2001). The Procrustes method scales and rotates the ordinations, using a minimum squared differences criterion. Then, the complement of the sum of the squared residuals between configurations in their optimal superimposition can be used as a measure of association (Gower, 1971). A permutation procedure (10 000 permutations) was used to assess the statistical significance of the Procrustes fit (Jackson, 1995).

The ordinations (PCo) generated for each covariance distance were compared with the ordinations (PCo) generated for the matrix of Euclidean distances between the mean shapes of each group, using Procrustes analysis (Gower, 1971; Peres-Neto \& Jackson, 2001). The Euclidean distances were calculated employing the different data sets described above and used in the different comparisons.

Finally, the degree of morphological integration in each treatment and ontogenetic stage was measured by the variance of eigenvalues (Wagner, 1990; Pavlicev et al. 2009). This measure of the degree of morphological integration is based on the fact that the eigenvalues of a matrix give the amount of variance associated with the corresponding eigenvector. If there are only a few eigenvalues that are large compared with the rest of the eigenvalues, then the variation of the characters is more or less confined to the few corresponding eigenvectors (Wagner, 1990). This analysis was made with the covariance matrix of 11 landmarks. We correct for differences in sample variance by scaling the variance of eigenvalues to the mean eigenvalue. In addition, to make the results presented here comparable to those in other studies, we report the relative eigenvalue variance (Pavlicev et al. 2009).

The statistical analyses were performed using R 2.8.0 ( $R$ Development Core Team, 2008), with the exception of the matrix correlations, which were performed in Mantel for Windows (Calvalcanti, 2008), and the random skewers analysis, which was performed using Skewers (Revell, 2007).

\section{Results}

Preliminary analyses indicated significant differences between Control and Sham groups, indicating that the surgery had an effect on the fetal growth. Consequently, Sham was used as the reference to analyze the specific effect of reduction in nutrient supply, independently of the surgery.

Figure 3 summarizes the mean values of size (CS) and their standard deviation by treatment and age. At the five ages analyzed, the IUGR group display lower mean values and larger standard deviations than the Sham group.

The ontogenetic trajectories of both groups are shown in the in shape space curve (Fig. 4). RW1 describes the dominant linear trend, which accounts for over half the variation 
in skull shape $(63.96 \%)$. The second component describes age-related deviations from the linear trend, but accounts for a small fraction of the variation (7.37\%). This component exhibits a non-linear relationship with age. In the Sham group scores on RW2 decrease with age from 5 to 13 days, then increase, whereas in the IUGR group scores on RW2 do not increase until the last age-class. In addition, Sham pups exhibit lesser dispersion than IUGR from 13 days, while in the previous age intervals both groups display a similar dispersion. Along the first component there are also

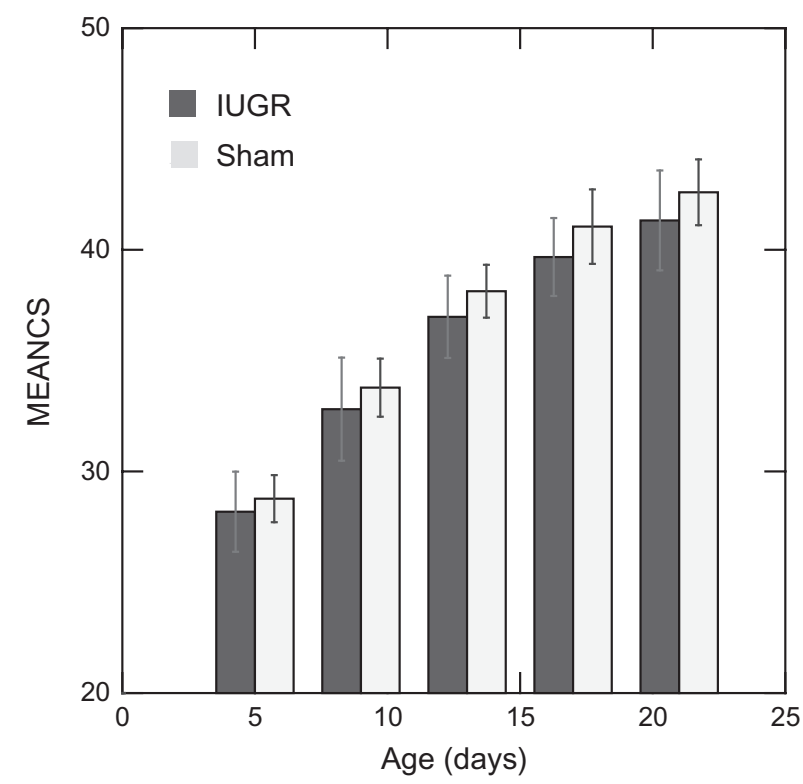

Fig. 3 Mean centroid size (MEANCS) by age and treatment. The error bars shown are standard deviations obtained through resampling the original datasets with replacement 1000 times. IUGR, intrauterine growth retardation. differences between treatments at each age interval. Such differences are more pronounced in younger individuals and decrease with age. RW1 displays a visually striking trend throughout ontogeny: skull elongation, to a greater extent anteriorly than posteriorly; the neurocranium becomes less globular and the flexion of the basicranium decreases (Fig. 4). The individual ontogenetic trajectories of both treatment groups differ in shape as well as in sizeshape space (Fig. 5a,b). The IUGR group shows more variation not only in the length along the first axis, but in the shape of individual trajectories.

The comparison of shape variance by Foote's disparity reveals that variance tends to decrease with age in both groups, IUGR and Sham (Fig. 6). This comparison also shows that while the IUGR group is more variable in almost all age intervals, the largest difference is found among 5-day-old specimens. Although we previously evaluated the measurement error, there is still some risk of imprecise identification of landmarks in very young animals (Bulygina et al. 2006). Thus, we evaluated how much error there would have to be in order to explain the difference in variance between the first and the older age-classes. We analyzed repeated measurements of the youngest and oldest group, and compared the amount of measurement error within each ageclass by analyzing the variance of Procrustes coordinates and the disparity between repeated measurements. The average Procrustes variance due to measurement error was 0.000032 for the 5 -day-old and 0.000024 for the 21 -day-old. The value of Foote's disparity was 0.0001 for the first ageclass and 0.0000001 for the last age-class. The disparity values of the youngest groups are two-eight times greater than the values of the older groups (Fig. 6). This means that even though measurement error in smaller animals is greater than in larger ones, it can not account for the reduction in within-group variance with age.

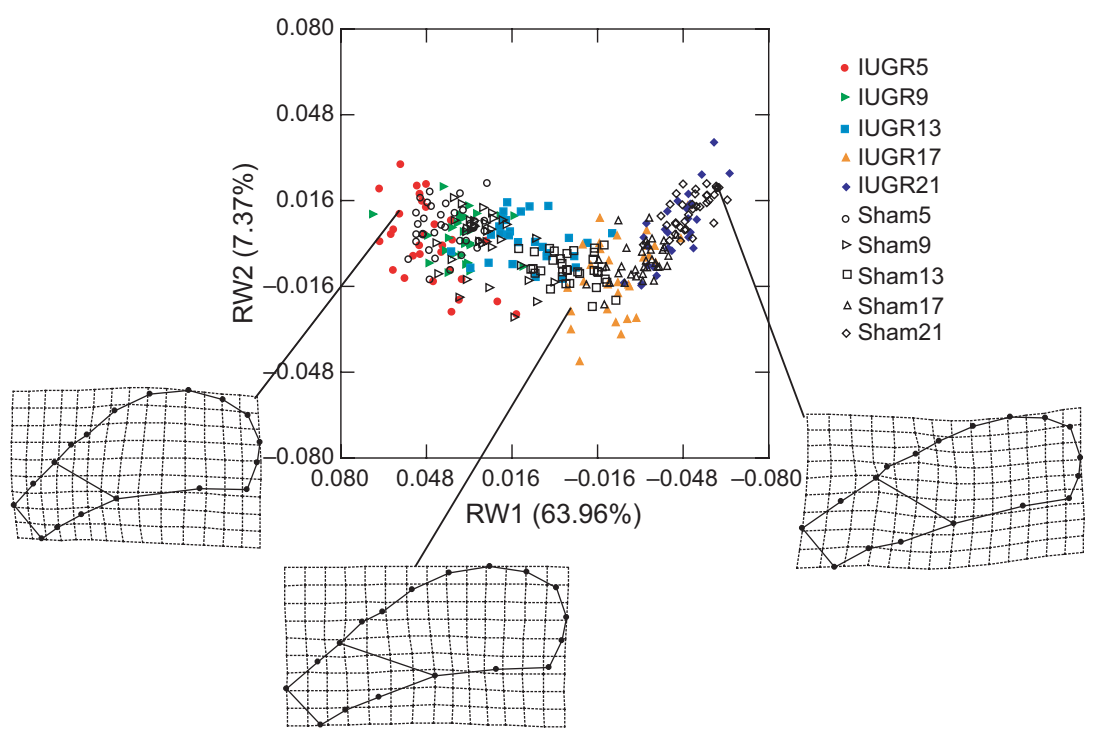

Fig. 4 First two relative warps (RW) estimated in base to the 18 coordinates of point data. The deformation grids show the shape changes between three age stages $(5,17$ and 21 days) and the consensus configuration along the first two RWs. IUGR, intrauterine growth retardation. 
Fig. 5 Individual ontogenetic trajectories in shape (a) and size-shape space (b) for IUGR (blue) and Sham (red) groups. In both graphs, specimens are ordered according to their age from left to right along the first axis. PC, principal component; RW, relative warp.
Fig. 6 Foote's disparity measurement by treatment and age. The bars represent the 95\% confidence intervals of each disparity value obtained by bootstrapping. IUGR, intrauterine growth retardation.
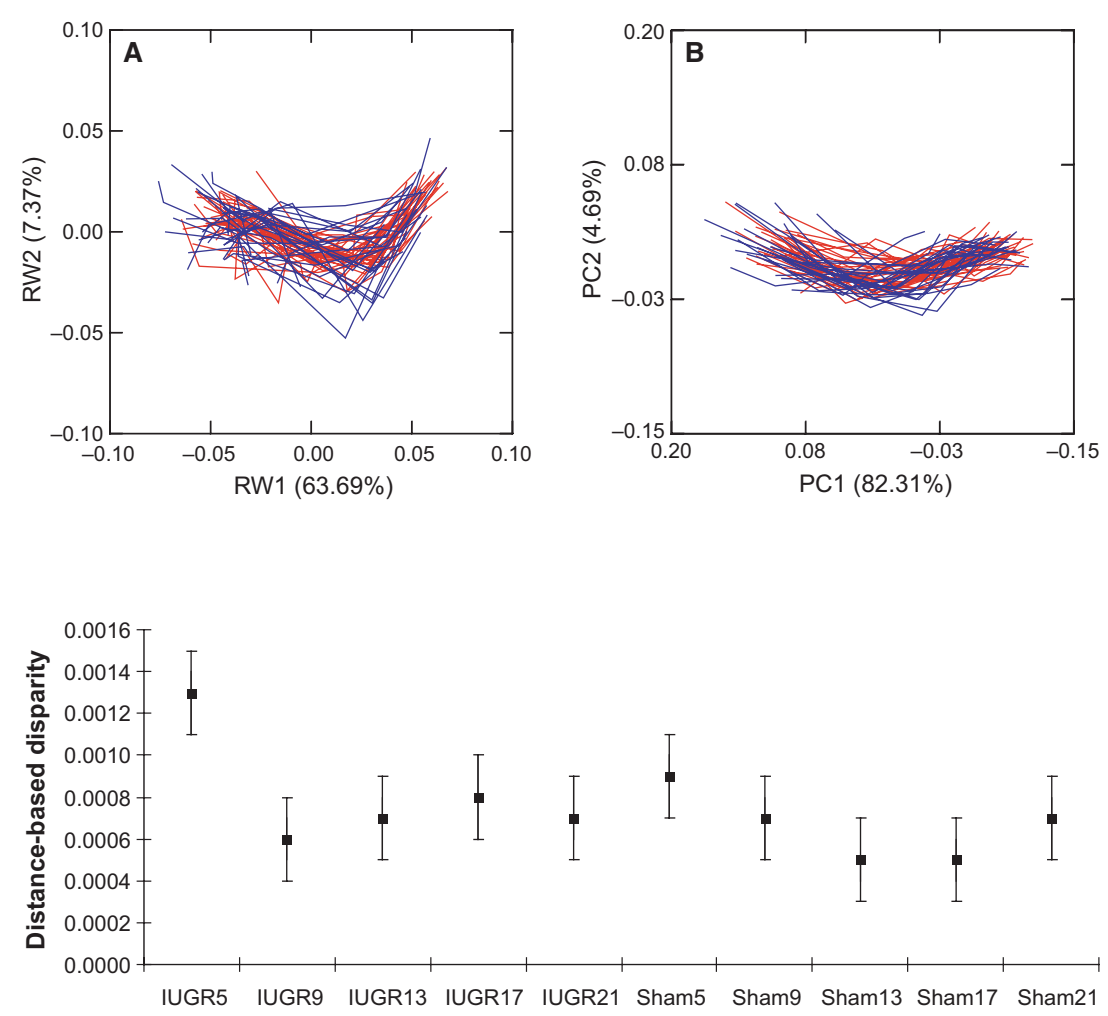

The matrix correlations between covariance matrices for each age within treatments obtained from 18 landmarks and semi-landmarks are presented in Table 2. Correlation values vary between 0.17 and 0.87 , and all are significant $(P \leq 0.01)$. Matrix repeatability ranges from 0.862 to 0.955 , with a mean of 0.918 , in the IUGR sample, and from 0.875 to 0.951 , with a mean of 0.904 , in the Sham sample. Adjusted matrix correlations between age groups range from 0.189 to 0.555 in the IUGR sample, and from 0.276 to 0.712 in the Sham sample (Table 2). In both samples the lowest value was obtained between the 5- and 21-day-old specimens, whereas the highest value was between 17- and 21-day-old specimens. The random skewers vector correlations between covariance matrices range between 0.45 and 0.86 , and all correlations are highly significant $(P \leq 0.01$; Table S1).

Figure 7a shows the principal coordinate ordination calculated using matrix correlation of the 18 landmarks and semi-landmarks, where points represent covariance matrices by treatment and age. Eigenvalues of the principal coordinate structure $(0.701,0.377,0.226,0.132)$ indicate that a $3 \mathrm{D}$ representation is sufficient, accounting for $78.27 \%$ of the total variation. Results of Procrustes test indicate a high correlation $\left(m_{12}=0.8847 ; P<0.001\right)$ between the space of the first three principal coordinates and the space of all PCos. The lines connecting the points represent the 'ontogenetic trajectory' of the covariance matrix for each treatment. PCo1 describes the dominant age trend, which accounts for around half the variation in skull shape $(42.10 \%)$. The PCo2 and $\mathrm{PCO} 3$ describe the differences between treatments; taken together they account for over $30 \%$ of the variation in skull shape. From 5 to 17 days, distances between covariance matrices of treatments at each age-class are large, and decrease at 21 days. So, at this age covariance matrices of Sham and IUGR groups exhibit greater similarity than in previous age-classes.

Because with 18 landmarks there are many degrees of freedom for the Procrustes shape coordinates in relation to the sample size, we repeated the analysis for the 11 principal components, accounting for $95 \%$ of the total variation. The matrix correlations between covariance matrices obtained from RWs are presented in Table 3. Correlation values vary between 0.009 and 0.67 , and only some of them are highly significant $(P \leq 0.01)$. Matrix repeatability ranges from 0.816 to 0.964 , with a mean of 0.894 , in the IUGR sample, and from 0.817 to 0.901 , with a mean of 0.866 , in the Sham sample. Adjusted matrix correlations between age groups range from 0.05 to 0.276 in the IUGR sample, and from -0.010 to 0.392 in the Sham sample (Table 3). The random skewers vector correlations range between 0.45 and 0.86 , and all results are significant $(P \leq 0.05$; Table $S 1)$.

To avoid the problem of having more variables than specimens, we also estimated the covariance matrices employing 11 coordinates of landmarks. Matrix correlations range between 0.13 and 0.61 (Table 4), and most of the results are highly significant $(P \leq 0.01)$.

Matrix repeatability ranges from 0.818 to 0.954 , with a mean of 0.886 , in the IUGR sample, and from 0.766 to 
8 Developmental plasticity in covariance structure of the skull, P. N. Gonzalez et al.

Table 2 Covariance matrix similarity for each pairwise age/treatment comparison, derived from the set of 18 landmarks and semi-landmarks.

\begin{tabular}{|c|c|c|c|c|c|c|c|c|c|c|}
\hline & IUGR5 & IUGR9 & IUGR13 & IUGR17 & IUGR21 & Sham5 & Sham9 & Sham13 & Sham 17 & Sham 21 \\
\hline IUGR5 & 0.915 & 0.509 & 0.379 & 0.365 & 0.189 & 0.501 & 0.622 & 0.323 & 0.291 & 0.194 \\
\hline IUGR9 & $\begin{array}{l}0.453^{*} \\
0.343-0.545\end{array}$ & 0.862 & 0.617 & 0.464 & 0.336 & 0.450 & 0.548 & 0.615 & 0.478 & 0.337 \\
\hline IUGR13 & $\begin{array}{l}0.346^{*} \\
0.223-0.459\end{array}$ & $\begin{array}{l}0.547^{*} \\
0.455-0.634\end{array}$ & 0.910 & 0.493 & 0.397 & 0.334 & 0.551 & 0.691 & 0.582 & 0.372 \\
\hline IUGR17 & $\begin{array}{l}0.340^{*} \\
0.241-0.429\end{array}$ & $\begin{array}{l}0.420^{*} \\
0.311-0.513\end{array}$ & $\begin{array}{l}0.457^{*} \\
0.368-0.542\end{array}$ & 0.950 & 0.554 & 0.358 & 0.559 & 0.331 & 0.487 & 0.499 \\
\hline IUGR21 & $\begin{array}{l}0.177 \\
-0.003-0.340\end{array}$ & $\begin{array}{l}0.305^{*} \\
0.187-0.418\end{array}$ & $\begin{array}{l}0.371^{*} \\
0.207-0.518\end{array}$ & $\begin{array}{l}0.528^{*} \\
0.428-0.616\end{array}$ & 0.955 & 0.246 & 0.283 & 0.287 & 0.676 & 0.917 \\
\hline Sham5 & $\begin{array}{l}0.460 * \\
0.378-0.534\end{array}$ & $\begin{array}{l}0.402^{*} \\
0.289-0.500\end{array}$ & $\begin{array}{l}0.306^{*} \\
0.213-0.405\end{array}$ & $\begin{array}{l}0.335^{*} \\
0.251-0.421\end{array}$ & $\begin{array}{l}0.231^{*} \\
0.148-0.312\end{array}$ & 0.923 & 0.488 & 0.335 & 0.422 & 0.276 \\
\hline Sham9 & $\begin{array}{l}0.558^{*} \\
0.406-0.670\end{array}$ & $\begin{array}{l}0.478^{*} \\
0.395-0.552\end{array}$ & $\begin{array}{l}0.493^{*} \\
0.372-0.603\end{array}$ & $\begin{array}{l}0.510^{*} \\
0.429-0.588\end{array}$ & $\begin{array}{l}0.259^{*} \\
0.075-0.447\end{array}$ & $\begin{array}{l}0.441^{*} \\
0.347-0.527\end{array}$ & 0.890 & 0.483 & 0.462 & 0.281 \\
\hline Sham13 & $\begin{array}{l}0.289 * \\
0.153-0.418\end{array}$ & $\begin{array}{l}0.535^{*} \\
0.458-0.604\end{array}$ & $\begin{array}{l}0.617^{*} \\
0.539-0.689\end{array}$ & $\begin{array}{l}0.302^{*} \\
0.193-0.403\end{array}$ & $\begin{array}{l}0.263^{*} \\
0.121-0.413\end{array}$ & $\begin{array}{l}0.301^{*} \\
0.208-0.386\end{array}$ & $\begin{array}{l}0.424^{*} \\
0.299-0.533\end{array}$ & 0.880 & 0.549 & 0.300 \\
\hline Sham17 & $\begin{array}{l}0.263^{*} \\
0.147-0.359\end{array}$ & $\begin{array}{l}0.420 * \\
0.320-0.510\end{array}$ & $\begin{array}{l}0.525^{*} \\
0.439-0.608\end{array}$ & $\begin{array}{l}0.448^{*} \\
0.341-0.538\end{array}$ & $\begin{array}{l}0.625^{*} \\
0.560-0.688\end{array}$ & $\begin{array}{l}0.383^{*} \\
0.290-0.471\end{array}$ & $\begin{array}{l}0.409 * \\
0.286-0.527\end{array}$ & $\begin{array}{l}0.486 * \\
0.403-0.566\end{array}$ & 0.892 & 0.712 \\
\hline Sham 21 & $\begin{array}{l}0.181 \\
-0.009-0.351\end{array}$ & $\begin{array}{l}0.305^{*} \\
0.156-0.401\end{array}$ & $\begin{array}{l}0.346^{*} \\
0.187-0.504\end{array}$ & $\begin{array}{l}0.474^{*} \\
0.367-0.572\end{array}$ & $\begin{array}{l}0.875^{*} \\
0.839-0.900\end{array}$ & $\begin{array}{l}0.259 * \\
0.177-0.344\end{array}$ & $\begin{array}{l}0.257^{*} \\
0.040-0.467\end{array}$ & $\begin{array}{l}0.274^{*} \\
0.139-0.416\end{array}$ & $\begin{array}{l}0.656^{*} \\
0.595-0.711\end{array}$ & 0.951 \\
\hline
\end{tabular}

Matrix repeatability $(t)$ is given in bold along the diagonal, with raw correlations below and adjusted correlations above. $95.0 \%$ confidence intervals for Mantel correlation estimated by bootstrap resampling are given under raw correlations. The numbers following the treatment name represent the age stage (in days). IUGR, intrauterine growth retardation. $* P<0.01$.

0.937 , with a mean of 0.862 , in the Sham sample. Adjusted matrix correlations between age groups range from 0.146 to 0.725 in the IUGR sample, and from 0.241 to 0.604 in the Sham sample (Table 4). The skewers vector correlations vary between 0.47 and 0.76 ( $P \leq 0.05$; Table S1).

Pairwise MB distances between the covariance matrices estimated based on the 11 principal components and 11 landmarks produced similar results (Table S2). Figure 7b shows PCo performed on the 11 principal component-based distances. The 3D representation accounted for $60.82 \%$ of the total variation. Results of Procrustes test indicate a high correlation $(0.7799 ; P<0.001)$ between the space of the first three principal coordinates and the space of all PCos. The ordination obtained is similar to that of the ordination obtained using matrix correlations of the 18 landmarks and semi-landmarks (Fig. 7a), showing the dominant age trend in PCo1 $(28.44 \%)$, and the differences between covariance matrices of each treatment at the different age-classes in the $\mathrm{PCO} 2$ and $\mathrm{PCO} 3$ (about $30 \%$ of the total variation). The distances between covariance matrices of treatments are large at the first age-classes and decrease at 21 days.

The Procrustes analyses of the principal coordinates calculated using matrix correlation, random skewers vector correlations and $\mathrm{MB}$ distances of the 11 landmarks, 18 landmarks and semi-landmarks, and the 11 principal components display strong and significant associations with each other (Table S3). This means that these methods lead to very similar results.
Finally, the Procrustes analysis of Euclidean distances against covariance distances (Table S4) shows a strong and significant association between them. These results demonstrate that when differences in mean values are more striking, the differences in covariance structure are also more prominent, being both affected by the factors age and treatments.

The IUGR group shows higher values of the scaled variance of eigenvalues than the Sham group, except at the age of 9 days (Fig. 8). This result indicates that such variance is concentrated on fewer eigenvectors (see also Table S5).

\section{Discussion}

Comparison of covariance matrices using different approaches based on overall measure of similarity (matrix correlation, random skewers vector correlation) and difference (MB distance) revealed that covariance structure of the skull changes significantly with age. The correlations between the covariance matrices of the first three ageclasses and 21 days attain very low values both in IUGR and Sham groups, which range between 0.129 and 0.371 for the set of 18 landmarks and semi-landmarks, and 0.129 and 0.317 for the set of 11 landmarks. The skewer vector correlation also shows that the greater the difference between age-classes, the lower the correlation value. Accordingly, the distance between covariance matrices increases with age. The phenotypic variance of the skull displays a tempo- 

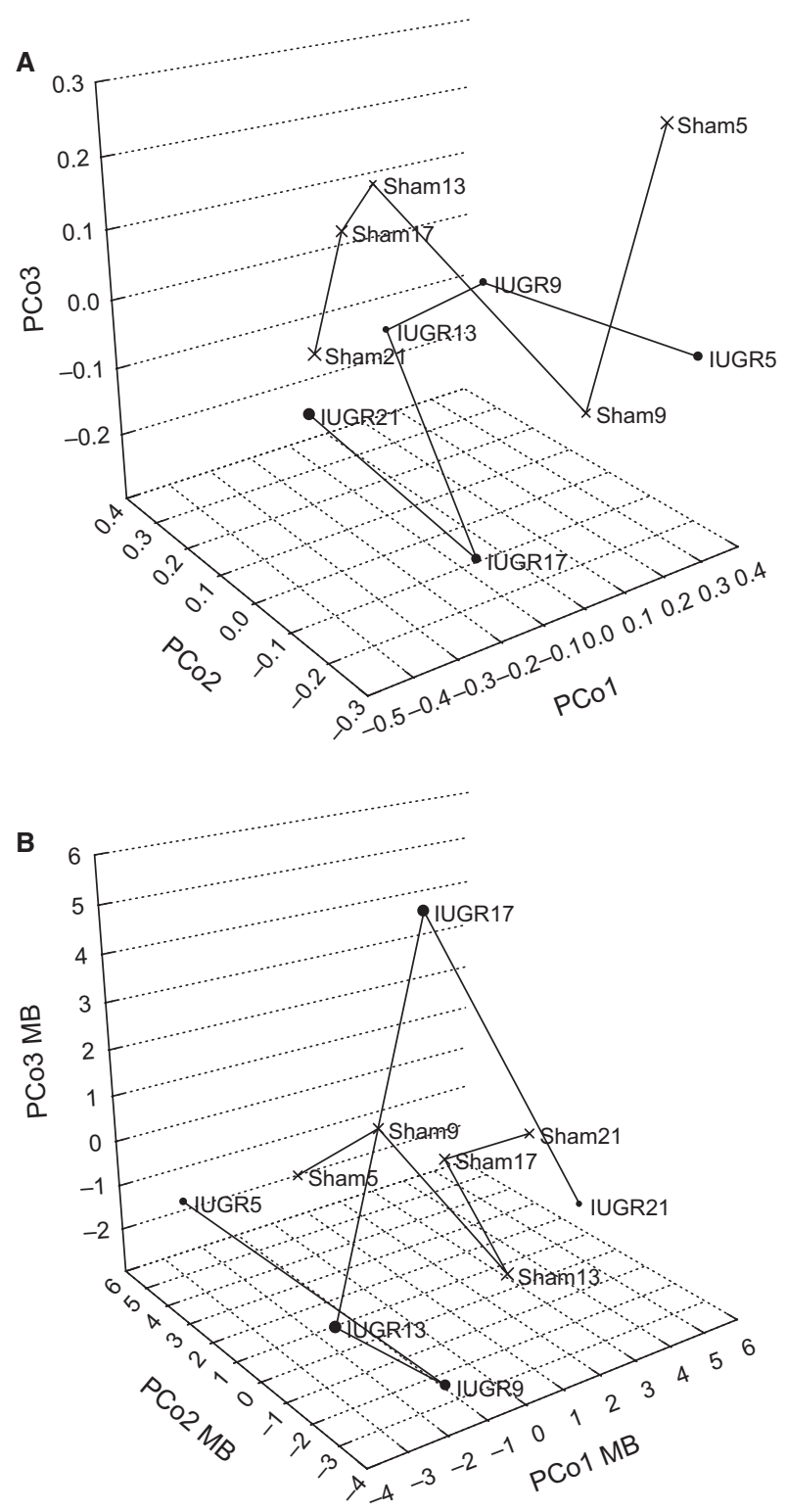

Fig. 7 (a) Principal coordinate ordination calculated using matrix correlation of the 18 landmarks and semi-landmarks. (b) Principal coordinate analysis (PCo) performed on MB distances between the covariance matrices estimated in base to the 11 principal components. IUGR, intrauterine growth retardation.

ral trend as well, with the highest values of distance-based disparity found at 5 days in both groups. After that age, the variance decreases significantly.

The changes observed in the variance-covariance structure with age are expected, as one fundamental characteristic of skeletal structures is that they are dynamic, changing continually over the lifetime of an individual. Throughout ontogeny, bone grows, reshapes and maintains itself by balancing production with removal of tissue, and thus it is expected that the dynamic of phenotypic variation of skeletal structures will change throughout ontogeny (Zelditch,
2005). Despite the recognized modifications of phenotypes from conception to adult life, the ontogenetic dynamics of variability in skeletal components has not been extensively studied, and most studies are centered on analyzing adult morphologies. Our results indicate a significant and rapid decrease of phenotypic variance early in postnatal growth. We argue that the pattern observed is an emergent property of developmental processes acting pre- and postnatally and not an artifact of the experiment conducted. In this study we analyzed two samples of 30 and 35 animals $X$-rayed at different ages from birth to weaning, in consequence we can rule out other factors that might alter the pattern of among-individual variance such as preservational artifacts, statistical vagaries of cross-sectional sampling and small sample size (Zelditch, 2005). These findings provide new and compelling evidence suggesting that variance in skull shape drops significantly early in postnatal growth, and support previous studies based on dried skeletal material as well as X-rays, which found that phenotypic variance diminished by about $50 \%$ in the first days of postnatal life (Nonaka \& Nakata, 1988; Riska et al. 1984; Hingst-Zaher et al. 2000; Zelditch et al. 2004; Zelditch, 2005). After weaning, phenotypic variance seems to be stable, suggesting that functional and developmental influences acting later on the skull have little effect on patterning their variance (Zelditch \& Carmichael, 1989; Willmore et al. 2006).

The causes of that pattern of reduction in among-individual variation with age, often referred to as the result of canalization, are not clear, and different hypotheses have been proposed to explain it. One hypothesis is that variation in growth produces variance in shape, and that targeted growth reduces the variance in shape (Zelditch, 2005). This is based on the idea that growth is self-stabilizing or targeted such that growth rates are adjusted so that an early retardation can be compensated by later acceleration to catch up to the norm. However, previous studies have found that variation in size accounts for a minor portion of the variation in skull shape, and thus targeted growth would be neither necessary nor sufficient to canalize shape (Zelditch et al. 2004; Zelditch, 2005). A second hypothesis, which does not require mechanisms that actively reduce that variation in timing, is that the high variance observed at early ages results from different rates of development and maturity among individuals at the same chronological age (Hallgrímsson, 1999). It is widely recognized that chronological and biological age do not match completely, and that important morphological changes are related to developmental events rather than age. For example, Monteiro \& Falconer (1966) showed that vaginal opening in mice occurs at different ages but at nearly the same weight. That means that if one compares individuals at the same age they will show large phenotypic variance, whereas if one considers some parameter of maturity (e.g. formation and fusion of epiphysis) it is expected they display lower variances. Variation in shape resulting from 


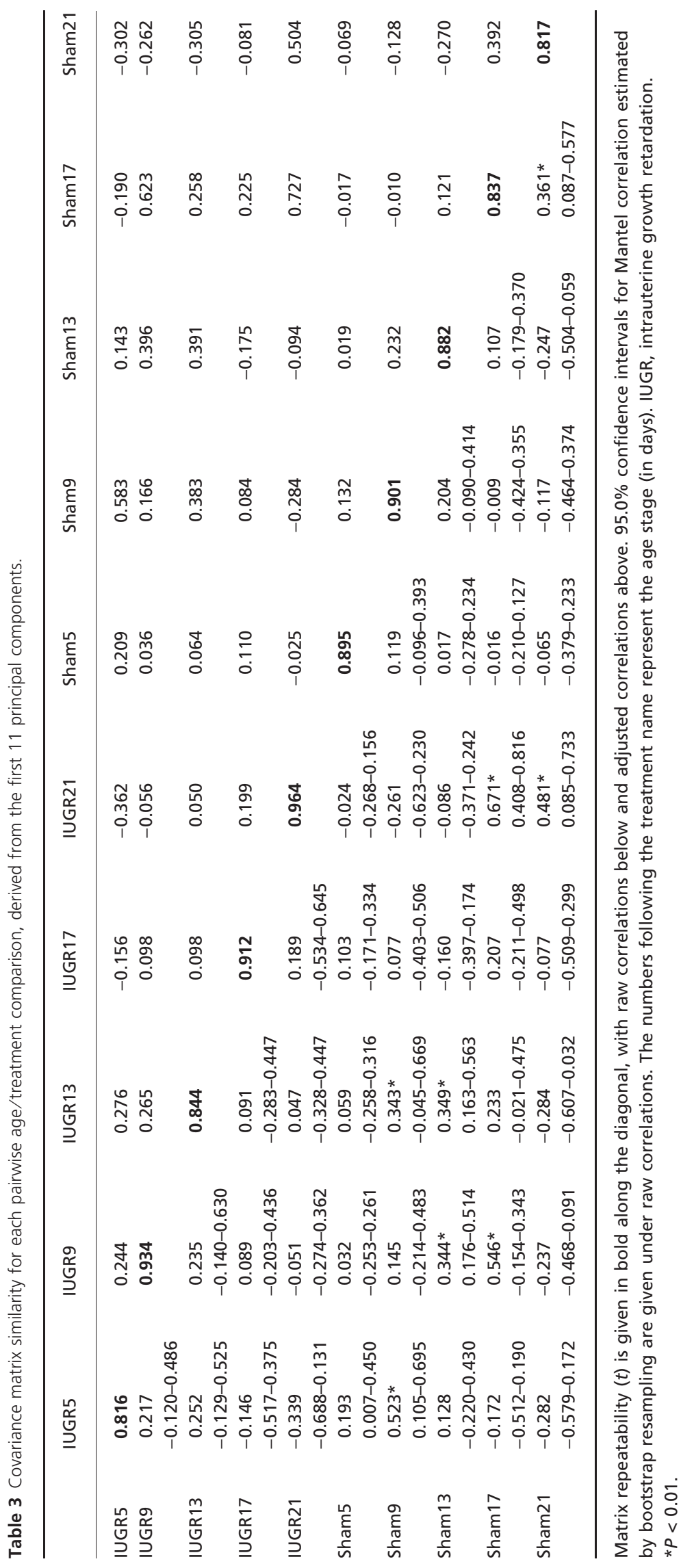


Table 4 Correlation between covariance matrixes for each pairwise age/treatment comparison, derived from the set of 11 landmarks.

\begin{tabular}{|c|c|c|c|c|c|c|c|c|c|c|}
\hline & IUGR5 & IUGR9 & IUGR13 & IUGR17 & IUGR21 & Sham5 & Sham 9 & Sham13 & Sham17 & Sham 21 \\
\hline IUGR5 & 0.818 & 0.725 & 0.521 & 0.418 & 0.146 & 0.552 & 0.776 & 0.487 & 0.360 & 0.199 \\
\hline \multirow[t]{2}{*}{ IUGR9 } & $0.613^{*}$ & 0.874 & 0.498 & 0.404 & 0.208 & 0.484 & 0.625 & 0.563 & 0.419 & 0.179 \\
\hline & $0.516-0.695$ & & & & & & & & & \\
\hline \multirow[t]{2}{*}{ IUGR13 } & $0.430 *$ & 0.424 * & 0.830 & 0.413 & 0.313 & 0.435 & 0.689 & 0.631 & 0.535 & 0.258 \\
\hline & $0.258-0.576$ & $0.294-0.556$ & & & & & & & & \\
\hline \multirow[t]{2}{*}{ IUGR17 } & $0.370 *$ & $0.369 *$ & $0.367 *$ & 0.953 & 0.441 & 0.387 & 0.608 & 0.235 & 0.399 & 0.262 \\
\hline & $0.261-0.476$ & $0.239-0.489$ & $0.248-0.475$ & & & & & & & \\
\hline \multirow[t]{2}{*}{ IUGR21 } & 0.129 & 0.190 & $0.279 *$ & 0.421 * & 0.955 & 0.239 & 0.275 & 0.227 & 0.624 & 0.622 \\
\hline & $-0.103-0.345$ & $0.025-0.344$ & $0.056-0.474$ & $0.285-0.536$ & & & & & & \\
\hline \multirow[t]{2}{*}{ Sham5 } & $0.473^{*}$ & $0.429 *$ & 0.376 * & $0.358 *$ & 0.221 * & 0.899 & 0.544 & 0.340 & 0.554 & 0.346 \\
\hline & $0.375-0.569$ & $0.286-0.553$ & $0.253-0.486$ & $0.246-0.457$ & $0.115-0.328$ & & & & & \\
\hline \multirow[t]{2}{*}{ Sham9 } & $0.615^{*}$ & 0.511 * & $0.549 *$ & $0.520 *$ & $0.236 *$ & 0.451 * & 0.766 & 0.601 & 0.557 & 0.351 \\
\hline & $0.449-0.727$ & $0.408-0.602$ & $0.407-0.691$ & $0.414-0.609$ & $-0.013-0.461$ & $0.338-0.563$ & & & & \\
\hline \multirow[t]{2}{*}{ Sham13 } & $0.414^{*}$ & $0.495^{*}$ & $0.540 *$ & $0.216^{*}$ & $0.209 *$ & $0.303 *$ & $0.495^{*}$ & 0.883 & 0.502 & 0.241 \\
\hline & $0.240-0.558$ & $0.390-0.589$ & $0.425-0.634$ & $0.062-0.354$ & $0.035-0.364$ & $0.181-0.415$ & $0.347-0.625$ & & & \\
\hline \multirow[t]{2}{*}{ Sham 17} & $0.296 *$ & $0.356 *$ & $0.444^{*}$ & $0.355^{*}$ & $0.554^{*}$ & $0.478^{*}$ & $0.443 *$ & $0.429 *$ & 0.827 & 0.604 \\
\hline & $0.134-0.437$ & $0.196-0.496$ & $0.309-0.565$ & $0.223-0.483$ & $0.469-0.636$ & $0.347-0.595$ & $0.284-0.586$ & $0.308-0.541$ & & \\
\hline \multirow[t]{2}{*}{ Sham 21} & 0.174 & 0.162 & 0.227 * & $0.248^{*}$ & $0.588^{*}$ & $0.317 *$ & 0.297 * & 0.220 * & $0.531 *$ & 0.937 \\
\hline & $-0.038-0.353$ & $0.006-0.298$ & $0.063-0.379$ & $0.106-0.384$ & $0.450-0.691$ & $0.211-0.422$ & $0.063-0.508$ & $0.091-0.337$ & $0.440-0.612$ & \\
\hline
\end{tabular}

Matrix repeatability $(t)$ is given in bold along the diagonal, with raw correlations below and adjusted correlations above. $95.0 \%$ confidence intervals for Mantel correlation estimated by bootstrap resampling are given under raw correlations. The numbers following the treatment name represent the age stage (in days). IUGR, intrauterine growth retardation.

$\star P<0.01$.

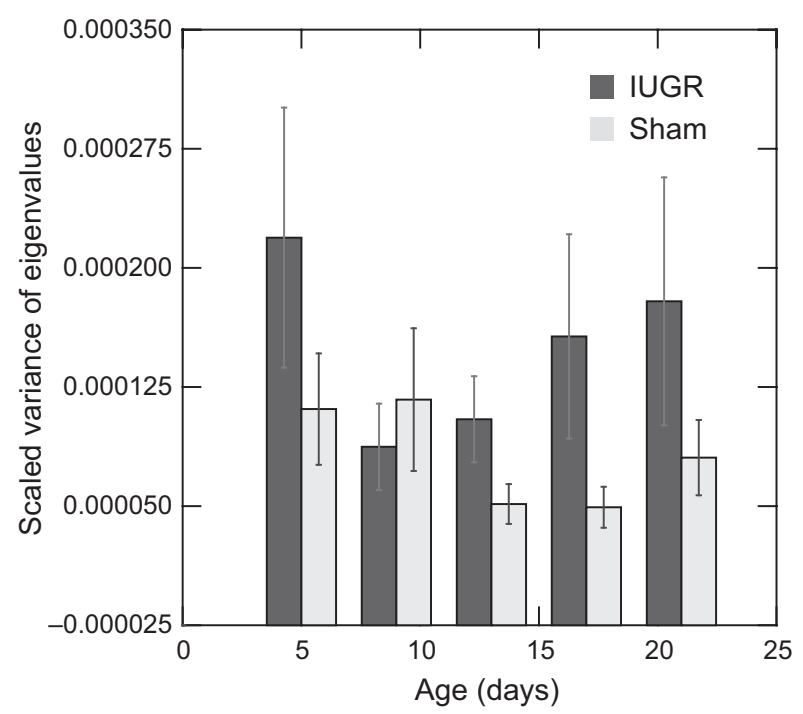

Fig. 8 Scaled variances of eigenvalues for intrauterine growth retardation (IUGR) and Sham groups by age. The error bars shown are standard deviations obtained through resampling the original datasets with replacement 1000 times.

variation in developmental timing should naturally decrease over time because very small differences in degree of maturity early in development have a far larger impact than they do later. These hypotheses as well as other alternatives require further research. Particularly the second hypotheses could be evaluated, for example, by analyzing the relationship between the degree of maturation and the morphological variation of the structures under study.

Our results suggest that the pattern of covariance is temporally dynamic from birth to 21 days, which is supported by the low correlations found between the youngest age-classes and the older. The values reported here are remarkably low, especially when they are contrasted with the values reported for comparison between covariance matrices of related species of rodents (Jamniczky \& Hallgrímsson, 2009) or even of two distantly related species, rhesus macaques and house mice (Hallgrímsson et al. 2004). Because only a few studies have explored the ontogenetic dynamic of covariance and there is no agreement on the techniques used either to describe skull shape or to compare covariance matrices, the results obtained by different authors are not easily compared. However, a significant difference in structure of variation with age has also been suggested by recent studies, which assert that the covariance matrices of skull change continually throughout the full period of postnatal development (Zelditch et al. 2006; Mitteroecker \& Bookstein, 2009).

The temporal dynamics of covariation structure of the skull might result from the influence of different developmental processes throughout ontogeny. During prenatal and early postnatal ontogeny the brain grew at a high rate, which became slower with age. As a consequence, a contrast is observed between neural and facial components; the precocial growth of the neurocranium contrast with the 
more retarded growth of the face (Zelditch et al. 1992). The deformation grids reflect such a temporal trend and show that the face became relatively elongated with respect to the neurocranium (Fig. 4). The grids also reveal remarkable shape changes in the neurocranium, which means that not only its relative size is modified with age. Particularly, the shape changes are characterized by a flattening of the neurocranium and modifications in the angle of the cranial base. A similar pattern of ontogenetic shape change has been described in rats, mice and other mammals (Moss \& Vilmann, 1978; Pucciarelli \& Dressino, 1996; Willmore et al. 2006). Our results suggest a strong ontogenetic allometry in the rat skull, which was confirmed by a multivariate regression of the Procrustes coordinates on log centroid size, which showed that size accounts for a large percentage of shape variation in both ontogenetic series $(44.727 \%$ for IUGR and $54.151 \%$ for Sham group). The allometric relationship between individual structures and size creates an axis of covariation that is often a dominant feature of the covariation structure of a population (Hallgrímsson et al. 2007). Thus, the allometric covariation produced by variation in size throughout ontogeny is one of the major determinants of cranial covariation structure. This is particularly relevant because if the influence of developmental processes responsible for regulating phenotypic variation varies in the course of ontogeny, and if variance and covariation structure also display a temporal variation, the age at which environmental and genetic factors act might strongly influence the possible evolutionary change in morphology.

The environmental perturbation provoked during pregnancy altered the skull growth, and reduced the mean size of the group under stress (Fig. 3). The IUGR group showed smaller size at the first age-class, which can be interpreted as a consequence of the reduced fetal growth. Such difference in size between treatments not only persisted throughout lactancy, when the mothers of both groups received a standard diet, but it became even larger. These findings support that environmental influences acting early in life play a fundamental role in the phenotypic outcome observed in late ontogeny. Increasing evidence suggests that the adult phenotype depends to a great extent on environmental signals operating during intrauterine development (a phenomena usually referred as intrauterine programming; Fowden et al. 2006). Changes in the intrauterine availability of nutrients, oxygen and hormones program tissue development, and can cause permanent structural and functional changes. Although most studies have focused on the consequences in physiological systems and the risk of developing some chronic diseases in adulthood, our findings suggest that this can be extended to morphological traits. The alteration of somatic growth, even when nutrient supply was restored, might indicate that some variation in growth-regulating factors that was induced by intrauterine nutritional status persists into postnatal life.
The alteration of growth did not affect equally all morphological traits observed here. The correlation values between covariance matrices of treatments at the same age were about 0.5 , being larger only for the last age-class. These values are very low, taking into account that the maximum correlation values were all above 0.85 . The covariation structure reflects the organization of organisms into sets of structures or traits that share developmental or genetic influences (Wagner et al. 2007; Hallgrímsson et al. 2009). If all traits were equally affected, we would not expect to find differences in covariance structure as other factors that could affect the covariance between traits, such as the genetic background and the postnatal environment, did not vary between treatments.

The reduction of nutrient supply during pregnancy also led to an increase in within-group variance of Procrustes coordinates, which is specially striking in the 5-day-old group (Fig. 6), as well as an increase in the variation of individual ontogenetic trajectories in shape and size-shape space (Fig. 5a,b). Such an increase in variance in the IUGR group was also related to an increase in integration, as measured by the scaled variance of eigenvalues. We hypothesized that if somatic growth has an important influence on covariance structure, phenotypic integration would increase. This prediction is based on the hypothesis that covariation is produced by variation in developmental processes that affect aspects of the phenotype unequally (Klingenberg, 2005; Hallgrímsson et al. 2007). If the variance of a process that is a major determinant of covariance in the ancestral population is increased, then integration will also increase because the proportion of the total variance explained by the axis of covariation that corresponds to this major developmental process will increase, leaving less variation to be explained by the less important factors (Hallgrímsson et al. 2007).

In summary, our findings suggest that the influence of environmental factors could be important in shaping the variance-covariance structure of phenotypic traits. Although phenotypic plasticity is commonly used in reference to individual traits to summarize how a given genotype (or population, or species) responds to a series of different environmental conditions by producing a more or less varied array of phenotypes, the same concept can be extended to trace the genotype-environment interaction in the amount and pattern of integration of complex phenotypes (Pigliucci et al. 1999; Pigliucci, 2004; Relyea, 2004). In this study we show that environmental perturbations can alter some fundamental developmental processes, which in turn result in a modification of variance-covariance structure of morphological phenotypes. The magnitude of such changes will depend on the strength of the environmental influence and the point in the life cycle in which it is produced (Badyaev, 2005).

The approach followed here also differs from most common studies of phenotypic plasticity, because we did not 
focus on adult form but on the plasticity along ontogenetic trajectories. This is particularly important because phenotypic plasticity in morphological traits is fundamentally a developmental phenomenon - where developmental pathways are expressed differently in response to specific environmental factors to produce continuously varying traits or discrete phenotypes (Frankino \& Raff, 2004). The lack of a developmental perspective can lead, for example, to interpret the production of invariant traits across environments as the developmental environment not affecting the final trait value or no developmental response to the environment, when actually they could be achieved through different developmental pathways and compensatory mechanisms that acted to buffer the environmental perturbation (Frankino \& Raff, 2004). In the absence of such developmental compensation, a different and less fit phenotype would be produced. According to our results, the structure of phenotypic covariance matrices of the two groups exposed to different environments was different in the first age-classes and then became more similar. This means that the ontogenetic trajectories differed as a consequence of the variation in environmental conditions. This reinforces the idea that measuring the adult phenotypes produced across a range of environments is necessary, but information about phenotypic changes throughout ontogeny is needed to understand the observed plastic responses.

\section{Acknowledgements}

This study was partially supported by grants from the Universidad Nacional de La Plata (UNLP) and Consejo Nacional de Investigaciones Científicas y Técnicas (CONICET). We thank Heather Jamniczky, Dan Lieberman and two anonymous reviewers for insightful comments.

\section{References}

Adams DC, Rohlf FJ, Slice DE (2004) Geometric morphometrics: ten years of progress following the 'revolution'. Ital J Zool 71, 5-16.

Allan GJ, Flint DJ, Patel K (2001) Insulin-like growth factor axis during embryonic development. Reproduction 122, 31-39.

Aubin-Horth N, Renn SC (2009) Genomic reaction norms: using integrative biology to understand molecular mechanisms of phenotypic plasticity. Mol Ecol 18, 3763-3780.

Badyaev AV (2005) Role of stress in evolution: from individual adaptability to evolutionary adaptation. In: Variation. A Central Concept in Biology (eds Hallgrímsson B, Hall BK), pp. 277-302. New York: Academic Press (Elsevier).

Bloomfield FH, Oliver MH, Harding JE (2006) The late effects of fetal growth patterns. Arch Dis Child Fetal Neonatal 91, 299304.

Bookstein FL (1991) Morphometric Tools for Landmark Data: Geometry and Biology. Cambridge: Cambridge University Press.

Bookstein FL (1997) Landmark methods for forms without landmarks: localizing group differences in outline shape. Med Image Anal 1, 225-243.
Bookstein FL, Streissguth AP, Sampson PD, et al. (2002) Corpus callosum shape and neuropsychological deficits in adult males with heavy fetal alcohol exposure. Neuroimage 15, 233-251.

Bulygina E, Mitteroecker P, Aiello L (2006) Ontogeny of facial dimorphism and patterns of individual development within one human population. Am J Phys Anthropol 131, 432-443.

Calvalcanti MJ (2008) Mantel for windows, Version 1.18. Freeware available at http://life.bio.sunysb.edu/morph/.

Cheverud JM (1996) Developmental integration and the evolution of pleiotropy. Am Zool 36, 44-50.

Dahinten S, Pucciarelli HM (1986) Variations in sexual dimorphism in the skulls of rats subjected to malnutrition, castration, and treatment with gonadal hormones. Am J Phys Anthropol 71, 63-67.

DeChiara TM, Efstratiadis A, Robertson EJ (1990) A growthdeficiency phenotype in heterozygous mice carrying an insulin-like growth factor II gene disrupted by targeting. Nature 345, 78-80.

Dressino V, Pucciarelli HM (1996) Efecto nutricional sobre el crecimiento craniofacial de Saimiri sciureus (Cebidae). Un estudio experimental. Rev Arg Antrop Biol 1, 98-112.

Fowden AL (2003) The insulin-like growth factors and fetoplacental growth. Placenta 24, 803-812.

Fowden AL, Giussani DA, Forhead AJ (2006) Intrauterine programming of physiological systems: causes and consequences. Physiology 21, 29-37.

Frankino WA, Raff RA (2004) Evolutionary importance and pattern of phenotypic plasticity: insights gained from development. In: Phenotypic Plasticity: Functional and Conceptual Approaches (eds DeWitt TJ, Scheiner SM), pp. 6481. New York: Oxford University Press.

Fu Q, Yu X, Callaway CW, et al. (2009) Epigenetics: intrauterine growth retardation (IUGR) modifies the histone code along the rat hepatic IGF-1 gene. FASEB J 23, 2438-2449.

Gilbert SF, Epel D (2009) Ecological Developmental Biology: Integrating Epigenetics, Medicine, and Evolution. Sunderland: Sinauer.

Gluckman PD, Hanson MA (2004) Living with the past: evolution, development, and patterns of disease. Science 305, 1733-1736.

Gower JC (1971) Statistical methods of comparing different multivariate analyses of the same data. In: Mathematics in the Archaeological and Historical Sciences (eds Hodson FR, Kendall DG, Tautu P), pp. 138-149. Edinburgh: Edinburgh University Press.

Hall BK (2003) Evo-devo: evolutionary developmental mechanisms. Int J Dev Biol 47, 491-495.

Hallgrímsson B (1999) Ontogenetic patterning of skeletal fluctuating asymmetry in rhesus macaques and humans: evolutionary and developmental implications. Int J Primatol 20, 121-151.

Hallgrímsson B, Lieberman DE (2008) Mouse models and the evolutionary developmental biology of the skull. Integr Comp Biol 48, 373-384.

Hallgrímsson B, Willmore K, Dorval C, et al. (2004) Craniofacial variability and modularity in macaques and mice. J Exp Zool (Mol Dev Evol) 302, 207-225.

Hallgrímsson B, Lieberman DE, Young NM, et al. (2007) Evolution of covariance in the mammalian skull. In: Tinkering - The Microevolution of Development (Novartis Found Symp 284), pp. 164-190. Chichester: John Wiley \& Sons Ltd. 
Hallgrímsson B, Jamniczky H, Young N, et al. (2009) Deciphering the palimpsest: studying the relationship between morphological integration and phenotypic covariation. Evol Biol 36, 355-376.

Hingst-Zaher E, Marcus L, Cerqueira R (2000) Application of geometric morphometrics to postnatal size and shape changes in the skull of Calomys expulsus. Hystrix, It J Mamm 11, 99-113.

Jackson DA (1995) PROTEST: a Procrustean randomization test of community environment concordance. Écoscience 2, 297303.

Jamniczky HA, Hallgrímsson B (2009) A comparison of covariance structure in wild and laboratory muroid crania. Evolution 63, 1540-1556.

Klingenberg CP (2005) Developmental constraints, modules and evolvability. In: Variation. A Central Concept in Biology (eds Hallgrímsson B, Hall BK), pp. 219-247. New York: Academic Press (Elsevier).

Le Roith D, Werner H, Beitner-Johnson D, et al. (1995) Molecular and cellular aspects of the insulin-like growth factor I receptor. Endocr Rev 16, 143-163.

Le Roith D, Bondy C, Yakar S, et al. (2001) The Somatomedin hypothesis: 2001. Endocr Rev 22, 53-74.

Marroig G, Cheverud JM (2001) A comparison of phenotypic variation and covariation patterns and the role of phylogeny, ecology, and ontogeny during cranial evolution of $\mathrm{New}$ World monkeys. Evolution 55, 2576-2600.

Martín MA, Serradas P, Ramos S, et al. (2005) Protein-caloric food restriction affects insulin-like growth factor system in fetal Wistar rat. Endocrinology 146, 1364-1371.

Miller JP, German RZ (1999) Protein malnutrition affects the growth trajectories of the craniofacial skeleton in rats. J Nutr 129, 2061-2069.

Mitteroecker P, Bookstein FL (2007) The conceptual and statistical relationship between modularity and morphological integration. Syst Bio/ 56, 818-836.

Mitteroecker P, Bookstein FL (2009) The ontogenetic trajectory of the phenotypic covariance matrix, with examples from craniofacial shape in rats and humans. Evolution 63, 727-737.

Mitteroecker P, Gunz P, Bernhard M, et al. (2004) Comparison of cranial ontogenetic trajectories among hominoids. J Hum Evol 46, 679-698.

Monteiro LS, Falconer DS (1966) Compensatory growth and sexual maturity in mice. Anim Prod 8, 179-192.

Moss ML, Vilmann H (1978) Studies on orthocephalization of the rat head. I. A model system for the study of adjustive cranial growth processes. Gegenbaurs Morphol Jahrb 124, 559-579.

Nijhout HF (2003) The control of growth. Development 130, 5863-5867.

Noble D (2002) The rise of computational biology. Nature 3, 460-463.

Nonaka K, Nakata M (1988). Genetic and environmental factors in the longitudinal growth of rats. III. Craniofacial shape change. J Craniofac Genet Dev Biology 8, 337-344.

Oyhenart EE, Orden B, Fucini MC, et al. (2003) Sexual dimorphism and postnatal growth of intrauterine growth retarded rats. Growth Dev Aging 67, 73-83.

Pavlicev M, Cheverud JM, Wagner GP (2009) Measuring morphological integration using eigenvalue variance. Evol Biol 36, 157-170.
Peres-Neto PR, Jackson DA (2001) How well do multivariate data sets match? The advantages of a Procrustean superimposition approach over the Mantel test. Oecologia 129, 169-178.

Perez SI, Bernal V, Gonzalez PN (2006) Differences between methods to sliding semi-landmarks: its importance in human population analyses. J Anat 208, 769-784.

Pigliucci M (2004) Studying the plasticity of phenotypic integration in a model organism. In: Phenotypic Integration. Studying the Ecology and Evolution of Complex Phenotypes (eds Pigliucci M, Preston K), pp. 155-175. New York: Oxford University Press.

Pigliucci M, Cammell K, Schmitt J (1999) Evolution of phenotypic plasticity a comparative approach in the phylogenetic neighbourhood of Arabidopsis thaliana. J Evol Biol 12, 779-791.

Pucciarelli HM, Dressino V (1996) Orthocephalization in the postweaning squirrel monkey. Am J Phys Anthropol 101, 173181.

R Development Core Team (2008) R: a language and environment for statistical computing. Vienna, Austria: $R$ Foundation for Statistical Computing, http://www.Rproject.org.

Relyea RA (2004) Integrating phenotypic plasticity when death is on the line: insights from predator-prey systems. In: Phenotypic Integration. Studying the Ecology and Evolution of Complex Phenotypes (eds Pigliucci M, Preston K), pp. 176190. New York: Oxford University Press.

Revell RJ (2007) Skewers software. Available at http:// anolis.oeb.harvard.edu / /iam/programs/.

Riska B, Atchley WR, Rutledge JJ (1984) A genetic analysis of targeted growth in mice. Genetics 107, 79-101.

Roff D (2000) The evolution of the G matrix: selection or drift? Heredity 84, 135-142.

Rohlf FJ (1993) Relative warps analysis and an example of its application to Mosquito wings. In: Contributions to Morphometrics (eds Marcus LF, Bello E, García-Valdecasas A), pp. 132-159. Madrid: Monografías del Museo Nacional de Ciencias Naturales.

Rohlf FJ (2007) tps serie softwares. Available at http// life.bio.sunysb.edu/morph/.

Rohlf FJ, Slice DE (1990) Extensions of the procrustes method for the optimal superimposition of landmarks. Syst Zool 39, 40-59.

Salazar-Ciudad I, Jernvall J, Newman SA (2003) Mechanisms of pattern formation in development and evolution. Development 130, 2027-2037.

Sheets HD (2003) IMP-Integrated Morphometrics Package. Buffalo, NY: Department of Physics, Canisius College.

Sultan SE, Stearns SC (2005) Environmentally contingent variation: phenotypic plasticity and norms of reaction. In: Variation. A Central Concept in Biology (eds Hallgrímsson B, Hall BK), pp. 303-332. New York: Academic Press (Elsevier).

Wagner GP (1990) A comparative study of morphological integration in Apis mellifera (Insecta, Hymenoptera). Z Zool Syst Evolut-forsch 28, 48-61.

Wagner GP, Pavlicev M, Cheverud J (2007) The road to modularity. Nat Genet 8, 921-931.

Willmore KE, Leamy L, Hallgrímsson B (2006) Effects of developmental and functional interactions on mouse cranial variability through late ontogeny. Evol Dev 8, 550-567. 
Zelditch ML (2005) Variation developmental regulation of variability. In: Variation. A Central Concept in Biology (eds Hallgrímsson B, Hall BK), pp. 249-276. New York: Academic Press (Elsevier).

Zelditch ML, Carmichael AC (1989) Ontogenetic variation in patterns of developmental and functional integration in skulls of Sigmodon fulviventer. Evolution 43, 814-824.

Zelditch ML, Bookstein FL, Lundrigan BL (1992) Ontogeny of integrated skull growth in the cotton rat Sigmodon fulviventer. Evolution 46, 1164-1180.

Zelditch ML, Lundrigan BL, Garland T (2004) Developmental regulation of skull morphology. I. Ontogenetic dynamics of variance. Evol Dev 6, 194-206.

Zelditch ML, Mezey J, Sheets HD, et al. (2006) Developmental regulation of skull morphology II: ontogenetic dynamics of covariance. Evol Dev 8, 46-60.

\section{Supporting Information}

Additional Supporting Information may be found in the online version of this article:
Table S1 Random skewers vector correlation for each pairwise age/treatment comparison employing 18 landmarks (above the diagonal) and 11 principal components.

Table S2 Mitteroecker-Bookstein distance for each pairwise age/treatment comparison.

Table S3 Procrustes values $\left(m_{12}\right)$ for pairwise comparison between matrix correlation (MC), random skewers vector correlations (RS) and MB distances estimated with the 11 landmarks (11L), 18 landmarks and semi-landmarks (18L) and 11 principal components (11RWs) data sets.

Table S4 Procrustes values $\left(m_{12}\right)$ of Euclidean distances between group mean shapes against the three covariance distances.

Table S5 Relative eigenvalue variance by age and treatment.

As a service to our authors and readers, this journal provides supporting information supplied by the authors. Such materials are peer-reviewed and may be re-organized for online delivery, but are not copy-edited or typeset. Technical support issues arising from supporting information (other than missing files) should be addressed to the authors. 